\title{
Application of Molecular Hydrogen as a Novel Antioxidant in Sports Science
}

\author{
Takuji Kawamura $\mathbb{D}^{1}{ }^{1}$ Kazuhiko Higashida, $^{2}$ and Isao Muraoka ${ }^{1}$ \\ ${ }^{1}$ Faculty of Sport Sciences, Waseda University, 2-579-15 Mikajima, Tokorozawa, Saitama 359-1192, Japan \\ ${ }^{2}$ Department of Food Science and Nutrition, The University of Shiga Prefecture, 2500, Hassaka-cho, Hikone, Shiga 522-8533, Japan \\ Correspondence should be addressed to Takuji Kawamura; tkawamura@aoni.waseda.jp
}

Received 28 September 2019; Revised 9 December 2019; Accepted 19 December 2019; Published 17 January 2020

Academic Editor: Ana Lloret

Copyright (c) 2020 Takuji Kawamura et al. This is an open access article distributed under the Creative Commons Attribution License, which permits unrestricted use, distribution, and reproduction in any medium, provided the original work is properly cited.

\begin{abstract}
Molecular hydrogen $\left(\mathrm{H}_{2}\right)$ is a colorless, tasteless, odorless, and minimal molecule with high flammability. Although $\mathrm{H}_{2}$ has been thought to be an inert gas in living bodies for many years, an animal study reported that inhalation of $\mathrm{H}_{2}$ gas decreased oxidative stress and suppressed brain injury caused by ischemia and reperfusion injury due to its antioxidant action. Since then, the antioxidant action of $\mathrm{H}_{2}$ has attracted considerable attention and many studies have reported on its benefits. Most studies have reported the effects of $\mathrm{H}_{2}$ on diseases such as cancer, diabetes, cerebral infarction, and Alzheimer's disease. However, little is known regarding its effects on healthy subjects and exercise. Thus far, including our study, only 6 studies have explored the effect of $\mathrm{H}_{2}$ on exercise. $\mathrm{H}_{2}$ is the smallest molecule and therefore can easily penetrate the cellular membrane and rapidly diffuse into organelles. $\mathrm{H}_{2}$ is thought to be able to selectively reduce hydroxyl radicals and peroxynitrite and does not affect physiologically reactive species. $\mathrm{H}_{2}$ can be supplied to the body through multiple routes of administration, such as oral intake of $\mathrm{H}_{2}$ water and $\mathrm{H}_{2}$ bathing. Therefore, $\mathrm{H}_{2}$ may be a potential alternative strategy for conventional exogenous antioxidant interventions in sports science. The purpose of this review is to provide evidence regarding the effects of $\mathrm{H}_{2}$ intake on changes in physiological and biochemical parameters, centering on exercise-induced oxidative stress, for each intake method. Furthermore, this review highlights possible future directions in this area of research.
\end{abstract}

\section{Introduction}

Molecular hydrogen $\left(\mathrm{H}_{2}\right)$ is a colorless, tasteless, odorless, and minimal molecule with high flammability [1]. Most mammals, including humans, do not synthesize hydrogenase, which is a catalyst for the activation of $\mathrm{H}_{2}$ [2], and therefore, $\mathrm{H}_{2}$ has long been considered to be an inert gas in mammalian cells. However, in 2007, a pioneer study reported that $\mathrm{H}_{2}$ can selectively reduce hydroxyl radicals $(\mathrm{OH})$ and peroxynitrite $\left(\mathrm{ONOO}^{-}\right)$in cultured cells but not other reactive species, such as superoxide $\left(\mathrm{O}_{2}^{--}\right)$, hydrogen peroxide $\left(\mathrm{H}_{2} \mathrm{O}_{2}\right)$, and nitric oxide (NO) [3]. The study also demonstrated that inhalation of $\mathrm{H}_{2}$ gas markedly decreased oxidative stress and suppressed brain injury caused by ischemia and reperfusion injury in rats [3]. Since the study was carried out, the amount of research on the antioxidant and therapeutic effects of $\mathrm{H}_{2}$ has increased rapidly. Moreover, studies have suggested that $\mathrm{H}_{2}$ can prevent the progression of various diseases $[1,4-8]$. As such, research regarding the medical applications of $\mathrm{H}_{2}$ is steadily progressing, with several clinical studies already started [1, 4-8].

In sports science, there is limited research regarding the antioxidant effect of $\mathrm{H}_{2}$ on exercise-induced oxidative stress. Unlike conventional antioxidants, $\mathrm{H}_{2}$ is a gas molecule and as such it is believed to have several advantages for application in sports science $[4,6,9]$ (Table 1 ). Firstly, $\mathrm{H}_{2}$ is the smallest molecule and thus can penetrate the cellular membrane and rapidly diffuse into organelles (e.g., mitochondria). Secondly, $\mathrm{H}_{2}$ is thought to have no effect on physiologically reactive species (e.g., $\mathrm{H}_{2} \mathrm{O}_{2}$ ), as it can selectively reduce $\mathrm{OH}$ and $\mathrm{ONOO}^{-}$. Finally, $\mathrm{H}_{2}$ can be supplied to the body through multiple routes of administration, such as oral intake of $\mathrm{H}_{2}$ water, $\mathrm{H}_{2}$ bathing, intravenous infusion of $\mathrm{H}_{2}$-saline, and inhalation of $\mathrm{H}_{2}$ gas. In addition to these advantages, $\mathrm{H}_{2}$ 
TABLE 1: Advantages and disadvantages of molecular hydrogen.

\begin{tabular}{l}
\hline Advantages \\
Easily penetrates the cellular membrane and rapidly diffuses into \\
organelles \\
Selectively reduces $\mathrm{OH}^{\mathrm{N}}$ and $\mathrm{ONOO}^{-}$and does not affect \\
physiological reactive species \\
Can be supplied to the body through multiple routes of \\
administration \\
Can be used with minimal side effects as it is excreted by \\
exhaling \\
Disadvantages \\
Dwells in the body for a short time \\
The optimal intake protocol has not been established \\
The effects of long-term intake are unknown \\
Small number of studies
\end{tabular}

can be used with minimal side effects as it is excreted by exhaling. Despite several disadvantages (Table 1), the aforementioned advantages of $\mathrm{H}_{2}$ use are expected to lead to an increase in research regarding its application in sports science.

The purpose of this review is to provide evidence regarding the effects of $\mathrm{H}_{2}$ intake on changes in physiological and biochemical parameters, centering on exercise-induced oxidative stress, as well as illustrate the mechanisms underlying the biological actions of $\mathrm{H}_{2}$. More specifically, this review describes findings from previous studies regarding the effects of each method of $\mathrm{H}_{2}$ administration. Moreover, we also summarize possible future directions for this area of research.

\section{Biological Actions of Molecular Hydrogen}

Although the antioxidative action of $\mathrm{H}_{2}$ was suggested in the study by Dole et al. [10] in 1975, its biological action has been overlooked for many years. Later, in 2007, it was reported that $\mathrm{H}_{2}$ selectively removes $\mathrm{OH}$ and $\mathrm{ONOO}^{-}$, which are strong oxidants, in vitro and that $\mathrm{H}_{2}$ suppresses oxidative stress after ischemia and reperfusion injury in vivo [3]. Since then, $\mathrm{H}_{2}$ has attracted widespread interest as a novel antioxidant and numerous previous studies have reported on the effectiveness of $\mathrm{H}_{2}$ for various diseases and disease models associated with oxidative stress [1, 4-8]. However, the direct removal of $\mathrm{OH}$ and $\mathrm{ONOO}^{-}$alone cannot fully explain the beneficial effects exerted by $\mathrm{H}_{2}$ in these diseases. Therefore, the indirect effects of $\mathrm{H}_{2}$ on the regulation of intracellular signaling pathways and gene expression have been investigated [1,4-8]. Specifically, it has been shown that $\mathrm{H}_{2}$ activates Nrf2 (nuclear factor-erythroid-derived 2-like-2) under oxidative stress conditions to increase the gene expression of antioxidant enzymes such as superoxide dismutase (SOD) and catalase $[1,4-8] . \mathrm{H}_{2}$ has also been shown to downregulate the transcription factor NF- $\kappa \mathrm{B}$ and inflammatory cytokines (e.g., interleukin- (IL-) $1 \beta$, IL-6, and tumor necrosis factor (TNF- $\alpha)$ ) in oxidative stress-induced inflammation [1, 4-8]. Moreover, recent studies have suggested that $\mathrm{H}_{2}$ suppresses lipid peroxidation associated with free radical chain reactions [11]

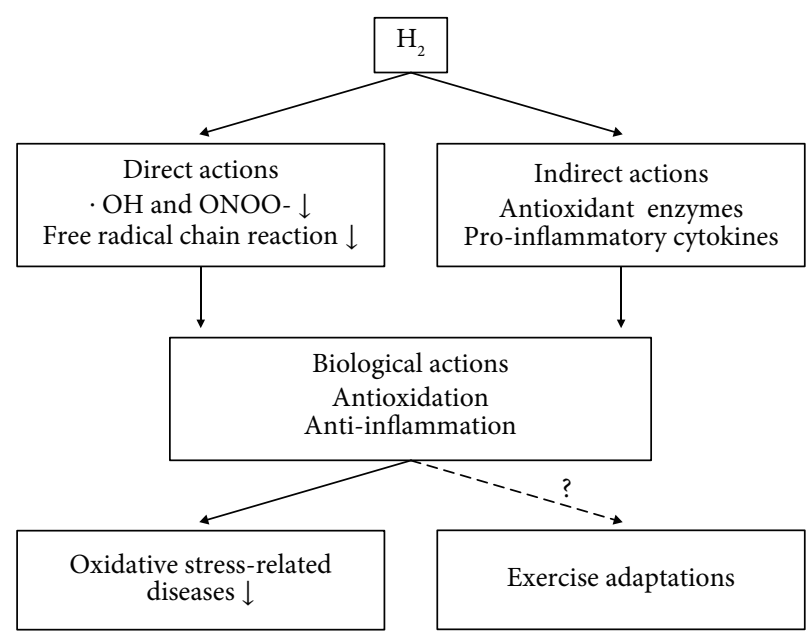

FIgURE 1: Possible simplified biological actions of molecular hydrogen: focusing on antioxidant and anti-inflammatory actions.

Taken together, the antioxidant action of $\mathrm{H}_{2}$ is considered to be not only direct, by selective removal of reactive species [3] and suppression of free radical chain reactions for lipid peroxidation [11], but also indirect, by inducing the expression of antioxidant enzymes. Furthermore, considering that $\mathrm{H}_{2}$ downregulates the expression of inflammatory cytokines [1,4-8], this may also suppress infiltration of phagocytes into the inflammatory site and subsequent release of reactive species. Possible biological actions of $\mathrm{H}_{2}$ are shown in Figure 1.

\section{Exercise-Induced Oxidative Stress}

Exercise is one of the physiological stimuli that promote the generation of reactive species in the living bodies. The generation of reactive species by exercise depends on exercise intensity, duration, and modality [12, 13]. The living body is equipped with an enzymatic or nonenzymatic antioxidant defense system. However, oxidative stress occurs when the levels of reactive species surpass the antioxidant capacity of the organism [14]. Exercise-induced oxidative stress has been shown to result in transient declines in physical functions through muscle fatigue, muscle damage and inflammation, and delayed-onset muscle soreness (DOMS) [12, 13, 15]. Moreover, there are many previous studies which have verified the effectiveness of taking exogenous antioxidants $[12,13,15]$.

On the other hand, it should be mentioned that longterm excessive intake of exogenous antioxidants inhibits redox-sensitive signaling pathways and interferes with physiological adaptations to exercise training, such as mitochondrial biogenesis, cardiac and skeletal muscle hypertrophy, and improvement of insulin sensitivity $[13,16]$. The results of previous studies regarding exercise redox biology indicate that the generation of excess levels of reactive species has a negative effect, while the generation of low-to-moderate levels of reactive species has a positive effect on the living body. The dependence of physiological responses or adaptations on the level of reactive species is called exercise 
TABLE 2: Effects of molecular hydrogen on exercise-induced oxidative stress, inflammation, and other indicators.

\begin{tabular}{|c|c|c|c|c|c|c|}
\hline Method & Study & Subjects & Intake protocol & Exercise & Markers & Effects \\
\hline \multirow{11}{*}{$\mathrm{H}_{2}$ water } & \multirow{4}{*}{ Aoki et al. [18] } & \multirow{4}{*}{$10 \mathrm{~T}$} & \multirow{4}{*}{$500 \mathrm{~mL} \times 3$ before $\mathrm{Ex}$} & \multirow{4}{*}{$\begin{array}{l}\text { Cycling }\left(75 \% \mathrm{VO}_{2} \max \right) \\
\text { Isokinetic knee extension }\end{array}$} & d-ROMs, BAP & $\rightarrow$ \\
\hline & & & & & $\mathrm{CK}$ & $\rightarrow$ \\
\hline & & & & & Lactate & $\downarrow$ \\
\hline & & & & & Peak torque & $\uparrow$ \\
\hline & \multirow{7}{*}{ Kawamura et al. [19] } & \multirow{7}{*}{32 rats } & \multirow{7}{*}{$\begin{array}{l}\text { Ad libitum } \\
14 \mathrm{~d} \text { before Ex }\end{array}$} & \multirow{7}{*}{ Running (exhaustion) } & TBARS & $\rightarrow$ \\
\hline & & & & & PC & $\rightarrow$ \\
\hline & & & & & TAC & $\rightarrow$ \\
\hline & & & & & Lactate & $\rightarrow$ \\
\hline & & & & & Glucose, FFA, TG & $\rightarrow$ \\
\hline & & & & & Muscle glycogen & $\rightarrow$ \\
\hline & & & & & Liver glycogen & $\uparrow$ \\
\hline \multirow{12}{*}{$\mathrm{H}_{2}$ bathing } & \multirow{7}{*}{ Kawamura et al. [21] } & \multirow{7}{*}{$9 \mathrm{UT}$} & \multirow{7}{*}{$\begin{array}{l}20 \mathrm{~min} \\
\text { Immediately and } 1-6 \mathrm{~d} \text { after } \\
\text { Ex }\end{array}$} & \multirow{7}{*}{$\begin{array}{l}\text { Downhill running } \\
\left(56 \% \mathrm{VO}_{2} \max \right)\end{array}$} & DOMS & $\downarrow$ \\
\hline & & & & & $\mathrm{CK}, \mathrm{Mb}$ & $\rightarrow$ \\
\hline & & & & & Lactate & $\rightarrow$ \\
\hline & & & & & TBARS & $\rightarrow$ \\
\hline & & & & & d-ROMs, BAP & $\rightarrow$ \\
\hline & & & & & MPO & $\rightarrow$ \\
\hline & & & & & IL-6, IL-17a & $\rightarrow$ \\
\hline & \multirow{5}{*}{ Kawamura et al. [22] } & \multirow{5}{*}{$9 \mathrm{UT}$} & \multirow{5}{*}{$\begin{array}{l}20 \text { min } \\
\text { Immediately and } 1-6 \mathrm{~d} \text { after } \\
\text { Ex }\end{array}$} & \multirow{5}{*}{$\begin{array}{l}\text { Downhill running } \\
\left(56 \% \mathrm{VO}_{2} \max \right)\end{array}$} & Total leukocytes & $\rightarrow$ \\
\hline & & & & & Neutrophils & $\rightarrow$ \\
\hline & & & & & Lymphocytes & $\rightarrow$ \\
\hline & & & & & $\mathrm{LmCL}$ & $\rightarrow$ \\
\hline & & & & & $\begin{array}{c}\text { Migratory } \\
\text { neutrophils }\end{array}$ & $\rightarrow$ \\
\hline \multirow{4}{*}{$\mathrm{H}_{2}$-saline } & \multirow{4}{*}{ Yamazaki et al. [23] } & \multirow{4}{*}{$\begin{array}{c}13 \\
\text { horses }\end{array}$} & \multirow{4}{*}{$\begin{array}{c}2 \mathrm{~L} \\
2 \mathrm{~h} \text { before Ex }\end{array}$} & \multirow{4}{*}{$\begin{array}{l}\text { High-intensity simulation } \\
\text { race }\end{array}$} & 8-OHdG & $\downarrow$ \\
\hline & & & & & d-ROMs, BAP & $\rightarrow$ \\
\hline & & & & & CK, AST, LDH & $\rightarrow$ \\
\hline & & & & & Lactate, uric acid & $\rightarrow$ \\
\hline \multirow{5}{*}{$\mathrm{H}_{2}$ gas } & \multirow{5}{*}{ Nogueira et al. [24] } & \multirow{5}{*}{60 rats } & \multirow{5}{*}{$\begin{array}{c}2 \% \cdot 2.4 \mathrm{~L} / \mathrm{min} \text { before and } \\
\text { during Ex }\end{array}$} & \multirow{5}{*}{ Running (80\% Vmax) } & TNF- $\alpha$, IL-6 & $\downarrow$ \\
\hline & & & & & SOD & $\uparrow$ \\
\hline & & & & & TBARS & $\downarrow$ \\
\hline & & & & & NOx & $\rightarrow$ \\
\hline & & & & & $\mathrm{p}$-CREB & $\downarrow$ \\
\hline
\end{tabular}

$\mathrm{H}_{2}$ water: oral intake of $\mathrm{H}_{2}$ water; $\mathrm{H}_{2}$-saline: intravenous infusion of $\mathrm{H}_{2}$-saline; $\mathrm{H}_{2}$ gas: inhalation of $\mathrm{H}_{2}$ gas; T: trained; UT: untrained; Ex: exercise; d-ROMs: diacron reactive oxygen metabolites; BAP: biological antioxidant potential; CK: creatine kinase; TBARS: thiobarbituric acid reactive substance; PC: protein carbonyl; TAC: total antioxidant capacity; FFA: free fatty acid; TG: triglyceride; DOMS: delayed-onset muscle soreness; Mb: myoglobin; MPO: myeloperoxidase; IL: interleukin; LmCL: luminol-dependent chemiluminescence; 8-OHdG: 8-hydroxydeoxyguanosine; AST: aspartate aminotransferase; LDH: lactate dehydrogenase; TNF- $\alpha$ : tumor necrosis factor- $\alpha$; SOD: superoxide dismutase; NOx: nitrite/nitrate; p-CREB: phosphorylation of cAMPresponsive element binding protein; $\uparrow:$ increase; $\rightarrow$ : no change; $\downarrow$ : decrease.

hormesis and may be an important criterion for the optimization of the effects of exogenous antioxidants [17].

\section{Research on the Application of $\mathrm{H}_{2}$ in Sports Science}

4.1. $\mathrm{H}_{2}$ Intake Methods. Six studies (Table 2) have been carried out on the effectiveness of $\mathrm{H}_{2}$ in sports science, involving 4 intake methods. Specifically, there are 2 studies on the oral intake of $\mathrm{H}_{2}$ water, 2 studies on $\mathrm{H}_{2}$ bathing, 1 study on intravenous infusion of $\mathrm{H}_{2}$-saline, and 1 study on the inhalation of
$\mathrm{H}_{2}$ gas. In this section, we introduce previous reports that have investigated the effects of each method of $\mathrm{H}_{2}$ administration on changes in physiological and biochemical parameters, centering on exercise-induced oxidative stress and inflammation.

4.2. Oral Intake of $\mathrm{H}_{2}$ Water. Intake of $\mathrm{H}_{2}$ water is one of the most practical and safe intake methods for daily life and in the sports field. $\mathrm{H}_{2}$ can be dissolved in water up to concentration of $0.8 \mathrm{mM}(1.6 \mathrm{mg} / \mathrm{L})$ under atmospheric pressure at room temperature [4]. However, in order to 
avoid a decrease in $\mathrm{H}_{2}$ concentration, it must be stored in an aluminum container.

Aoki et al. initially reported that oral intake of $\mathrm{H}_{2}$ water has no effect on blood reduction/oxidation (redox) biomarkers such as diacron reactive oxygen metabolites (d-ROMs) and biological antioxidant potential (BAP) but suppresses the elevation of blood lactate concentrations and reduces peak torque during exercise in trained young men [18]. Our research group investigated the effects of oral intake of $\mathrm{H}_{2}$ water on exercise-induced oxidative stress and its related indicators using an animal model [19]. Similar to the results of Aoki et al. [18], our findings indicated that a 2-week intake of $\mathrm{H}_{2}$ water did not affect redox homeostases, such as thiobarbituric acid reactive substances (TBARS), protein carbonyl (PC), and total antioxidant capacity (TAC), in both plasma and skeletal muscle during exhaustive running in fasting rats [19]. In addition, we also demonstrated that $\mathrm{H}_{2}$ water intake did not affect blood energy substrates, muscle glycogen content, and performance level, while it slightly suppressed liver glycogen utilization during exercise. In contrast to these results, our latest data showed that a 2-week intake of $\mathrm{H}_{2}$ water increases plasma lactate and free fatty acid concentrations, as well as liver glycogen utilization, during constant exercise at low intensity in fed rats (unpublished data).

Taken together, there is no report regarding the effectiveness of $\mathrm{H}_{2}$ water intake against exercise-induced oxidative stress and inflammation in humans and animals [18, 19]. On the other hand, there are fragmented reports on the possible effects of $\mathrm{H}_{2}$ intake on glucose metabolism [18], liver glycogen utilization [19], and performance levels [18] during exercise.

4.3. $\mathrm{H}_{2}$ Bathing. Aside from the oral intake of $\mathrm{H}_{2}$ water, $\mathrm{H}_{2}$ bathing is another method with high applicability in sports. For this method, an $\mathrm{H}_{2}$-producing agent is generally used (e.g., $\mathrm{MgH}_{2}$ ). The $\mathrm{H}_{2}$-producing agent can be stably stored for long periods and can be used safely and easily. The generated $\mathrm{H}_{2}$ is delivered into the body orally and transcutaneously, and it is considered that $\mathrm{H}_{2}$ reaches the whole body only 10 min after the $\mathrm{H}_{2}$ bath, based on the concentration of $\mathrm{H}_{2}$ in the breath [20].

Our research group investigated the influences of weekly $\mathrm{H}_{2}$ bathing on exercise-induced oxidative stress and inflammatory responses, as well as muscle damage and DOMS after downhill running [21]. Our findings showed that weekly $\mathrm{H}_{2}$ bathing had no influence on redox homeostasis (i.e., TBARS, d-ROMs, and BAP), inflammatory responses (i.e., IL-6, IL-17a, and myeloperoxidase (MPO)), and the degree of muscle damage markers (i.e., creatine kinase $(\mathrm{CK})$ and myoglobin $(\mathrm{Mb})$ ) in the blood. However, weekly $\mathrm{H}_{2}$ bathing alleviated DOMS as evaluated by the visual analogue scale 24 and $48 \mathrm{~h}$ after downhill running. We also investigated the effects of weekly $\mathrm{H}_{2}$ bathing on neutrophil dynamics and function, which play an important role in secondary oxidative stress after eccentric exercise [21]. Our results showed that $\mathrm{H}_{2}$ bathing after downhill running did not influence the peripheral neutrophil count or its functions, such as migration activity and reactive oxygen species
(ROS) productivity, as evaluated by luminol-dependent chemiluminescence (LmCL).

As described above, there is no conclusive evidence regarding the effectiveness of $\mathrm{H}_{2}$ bathing against muscle damage, secondary oxidative stress, and inflammation after eccentric exercise [21, 22]. Moreover, although the associated mechanism of action has not been elucidated, our results have shown that $\mathrm{H}_{2}$ may alleviate DOMS after eccentric exercise [21].

4.4. Intravenous Infusion of $\mathrm{H}_{2}$-Saline. Intravenous infusion of $\mathrm{H}_{2}$ saline is a method that can rapidly supply a large amount of $\mathrm{H}_{2}$ into the living body. However, this method may be difficult to use in the sports field due to its invasiveness.

Yamazaki et al. investigated the effects of an intravenous infusion of $\mathrm{H}_{2}$-saline on blood redox and metabolic/injury biomarkers in thoroughbred horses after a high-intensity simulation race [23]. Their results showed that an intravenous infusion of $\mathrm{H}_{2}$-saline decreased serum 8-hydroxydeoxyguanosine (8-OHdG), which reflects the development of DNA damage. However, there was no significant difference in the level of other redox (i.e., d-ROMs and BAP) and metabolic/injury biomarkers (e.g., lactate, uric acid, and CK) in the blood after the simulation race. Therefore, there is limited evidence regarding the efficacy of $\mathrm{H}_{2}$-saline infusion.

4.5. Inhalation of $\mathrm{H}_{2}$ Gas. From the viewpoint of experts in sports science, the inhalation of $\mathrm{H}_{2}$ gas is not a versatile method of $\mathrm{H}_{2}$ administration. However, this method can quickly supply a large amount of $\mathrm{H}_{2}$ to the living body. $\mathrm{H}_{2}$ gas can easily be inhaled through a ventilator circuit, face mask, or nasal cannula, and there is no risk of explosion when the concentration in the air is below $4 \%$ [20].

Nogueira et al. reported that inhalation of $2 \% \mathrm{H}_{2}$ gas while treadmill running suppresses TBARS levels and inflammatory biomarkers, such as TNF- $\alpha$ and IL-6, in rat plasma immediately or $3 \mathrm{~h}$ after exercise [24]. In addition, plasma SOD activity 3 hours after exercise was enhanced by $\mathrm{H}_{2}$ intake. This previous study also showed that the phosphorylation of skeletal muscle cAMP-responsive element binding (CREB) protein, which is involved in increasing oxidative metabolism and mitochondrial biogenesis, is attenuated by the inhalation of $\mathrm{H}_{2}$ gas at $3 \mathrm{~h}$ after exercise. Therefore, the inhalation of $\mathrm{H}_{2}$ gas simultaneously with exercise is effective in suppressing exercise-induced oxidative stress and inflammation but may inhibit the adaptation of skeletal muscle by exercise training.

4.6. Summary of the Results of Previous Studies. Although the effectiveness of oral intake of $\mathrm{H}_{2}$ water $[18,19]$ and $\mathrm{H}_{2}$ bathing $[21,22]$ has not been demonstrated, intravenous infusion of $\mathrm{H}_{2}$-saline [23] and inhalation of $\mathrm{H}_{2}$ gas [24] have been reported to suppress exercise-induced oxidative stress and/or inflammation. However, the antioxidant and anti-inflammatory effects of $\mathrm{H}_{2}$ intake have been observed only in animals $[23,24]$, and these effects have not been confirmed in humans regardless of the administration 
route $[18,19,21,22]$. Regarding the timing of $\mathrm{H}_{2}$ administration, it seems to be most effective before [23] or simultaneously with exercise [24].

Other than its antioxidant and anti-inflammatory effects, some studies have partly shown that $\mathrm{H}_{2}$ intake exerts some effects such as improvement of exercise performance [18], changes in glucose metabolism [18] and liver glycogen utilization [19], and alleviation of DOMS [21]. However, it should be mentioned that, like other antioxidants, $\mathrm{H}_{2}$ intake may partially inhibit physiological adaptations induced by exercise training [24].

\section{Future Directions}

Research on $\mathrm{H}_{2}$ for applications in sports science is in its incipient stages. Given the advantages of $\mathrm{H}_{2}$ (Table 1), it may be worth investigating the effects of $\mathrm{H}_{2}$ intake on physiological and biochemical responses, especially exerciseinduced oxidative stress and inflammation.

Firstly, as a future effort, it will be necessary to establish an optimal $\mathrm{H}_{2}$ intake protocol based on the dynamics of $\mathrm{H}_{2}$ in the body. In particular, among the methods of $\mathrm{H}_{2}$ intake, oral intake of $\mathrm{H}_{2}$ water and $\mathrm{H}_{2}$ bathing seem to be the most practical intake methods that can be used even in the general sports field. To date, few papers have reported changes in $\mathrm{H}_{2}$ concentration in the living body after $\mathrm{H}_{2}$ water intake and $\mathrm{H}_{2}$ bathing $[20,25]$. This suggests that either method of administration peaks in vivo between 5 and 10 minutes and then returns to baseline values by 60 minutes [20,25]. Therefore, in order to obtain an acute $\mathrm{H}_{2}$ effect, administration immediately before or during exercise may be more effective. As such, when verifying the acute effect, oral intake of $\mathrm{H}_{2}$ water may be more practical than $\mathrm{H}_{2}$ bathing. Since the effects of $\mathrm{H}_{2}$ are influenced by various factors, such as intake method, timing, concentration, dose, and frequency, it is of great importance to steadily accumulate evidence regarding the effects of $\mathrm{H}_{2}$ on reducing exercise-induced oxidative stress and inflammation.

Secondly, in addition to the $\mathrm{H}_{2}$ intake protocol, it is also necessary to conduct a study that takes into account the individual redox properties of the subjects. In recent years, the importance of personalized antioxidant strategies has been proposed [26]. Specifically, beneficial effects such as reducing exercise-induced oxidative stress and improving exercise performance due to ingestion of exogenous antioxidants are only seen in subjects with insufficient resting antioxidant levels, but these beneficial effects have not been observed in subjects with appropriate antioxidant levels at rest [27, 28]. Therefore, it is required to examine the effectiveness of $\mathrm{H}_{2}$ intake on exercise-induced oxidative stress and related indicators after screening the resting antioxidant status of the subjects.

Thirdly, the effects of long-term $\mathrm{H}_{2}$ intake on exercise adaptations must be clarified. As mentioned above, longterm and excessive antioxidant (e.g., vitamin $\mathrm{C}$ and vitamin E) intake has been shown to inhibit redox-sensitive signaling pathways and interfere with physiological adaptations to exercise training $[13,16]$. Although several studies have presented counterevidence $[29,30]$, long-term and excessive exogenous antioxidant intake should be avoided during exercise training. Unlike conventional antioxidants, $\mathrm{H}_{2}$ has been postulated to selectively reduce $\mathrm{OH}$ and $\mathrm{ONOO}^{-}$and not affect physiologically reactive species. However, previous studies have shown [24] that the phosphorylation of skeletal muscle CREB is attenuated after exercise by the inhalation of $\mathrm{H}_{2}$ gas. This suggests that $\mathrm{H}_{2}$ may interfere with the beneficial effects of exercise training by inhibiting cellular signaling pathways during acute exercise stimulus. Therefore, it is important to clarify the effect of chronic $\mathrm{H}_{2}$ intake on physiological adaptations induced by long-term exercise training (Figure 1).

Fourthly, it will be necessary to unravel the alternative mechanism underlying the antioxidant action of $\mathrm{H}_{2}$. Previous studies have shown that $\mathrm{H}_{2}$ intake is effective in improving exercise performance [18], changing the glucose metabolism and liver glycogen utilization $[18,19]$, and mitigating DOMS [21] without affecting redox biomarkers. On the other hand, several studies have investigated the effects of $\mathrm{H}_{2}$ on the buffer capacity of the blood, exhaled gas parameters, and exercise performance without measuring redox biomarkers. As such, there is only fragmented evidence regarding the positive effects of $\mathrm{H}_{2}$ intake [31-38]. Notably, many findings regarding the medical efficacy of $\mathrm{H}_{2}$ could not be explained solely by selective removal of reactive species [3] and suppression of free radical chain reactions for lipid peroxidation [11]. However, research on the action mechanism of $\mathrm{H}_{2}$ has just started, and further research development is expected in the future.

\section{Conclusions}

Here, we briefly summarized the current findings regarding the effect of $\mathrm{H}_{2}$ intake on changes in physiological and biochemical parameters, centering on exercise-induced oxidative stress. However, presently, there are few studies aimed at applying $\mathrm{H}_{2}$ in sports science and its effectiveness and long-term effects have not been fully demonstrated. Therefore, it is premature to conclude its usefulness. Since $\mathrm{H}_{2}$ is a gas molecule and has several advantages, it is worthwhile to continue research towards the application of $\mathrm{H}_{2}$ in sports science.

\section{Conflicts of Interest}

The authors declare that there is no conflict of interest regarding the publication of this paper.

\section{Acknowledgments}

We would like to thank Editage (https://www.editage.jp/) for English language editing. This work was supported by a subsidy system for academic papers Waseda University.

\section{References}

[1] C. S. Huang, T. Kawamura, Y. Toyoda, and A. Nakao, "Recent advances in hydrogen research as a therapeutic medical gas," Free Radical Research, vol. 44, no. 9, pp. 971-982, 2010. 
[2] J. Fritsch, O. Lenz, and B. Friedrich, "Structure, function and biosynthesis of $\mathrm{O}_{2}$-tolerant hydrogenases," Nature Reviews Microbiology, vol. 11, no. 2, pp. 106-114, 2013.

[3] I. Ohsawa, M. Ishikawa, K. Takahashi et al., "Hydrogen acts as a therapeutic antioxidant by selectively reducing cytotoxic oxygen radicals," Nature Medicine, vol. 13, no. 6, pp. 688694, 2007.

[4] Y. Hong, S. Chen, and J. M. Zhang, "Hydrogen as a selective antioxidant: a review of clinical and experimental studies," The Journal of International Medical Research, vol. 38, no. 6, pp. 1893-1903, 2010.

[5] B. J. Dixon, J. Tang, and J. H. Zhang, "The evolution of molecular hydrogen: a noteworthy potential therapy with clinical significance," Medical Gas Research, vol. 3, no. 1, p. 10, 2013.

[6] S. Ohta, "Molecular hydrogen as a preventive and therapeutic medical gas: initiation, development and potential of hydrogen medicine," Pharmacology \& Therapeutics, vol. 144, no. 1, pp. 1-11, 2014.

[7] M. Ichihara, S. Sobue, M. Ito, M. Ito, M. Hirayama, and K. Ohno, "Beneficial biological effects and the underlying mechanisms of molecular hydrogen - comprehensive review of 321 original articles," Medical Gas Research, vol. 5, no. 1, 2015.

[8] L. Ge, M. Yang, N. N. Yang, X. X. Yin, and W. G. Song, "Molecular hydrogen: a preventive and therapeutic medical gas for various diseases," Oncotarget, vol. 8, no. 60, pp. 102653-102673, 2017.

[9] S. M. Ostojic, "Molecular hydrogen in sports medicine: new therapeutic perspectives," International Journal of Sports Medicine, vol. 36, no. 4, pp. 273-279, 2015.

[10] M. Dole, F. R. Wilson, and W. P. Fife, "Hyperbaric hydrogen therapy: a possible treatment for cancer," Science, vol. 190, no. 4210, pp. 152-154, 1975.

[11] K. Iuchi, A. Imoto, N. Kamimura et al., "Molecular hydrogen regulates gene expression by modifying the free radical chain reaction-dependent generation of oxidized phospholipid mediators," Scientific Reports, vol. 6, no. 1, article 18971, 2016.

[12] K. Fisher-Wellman and R. J. Bloomer, "Acute exercise and oxidative stress: a 30 year history," Dynamic Medicine, vol. 8, no. $1,2009$.

[13] T. Kawamura and I. Muraoka, "Exercise-induced oxidative stress and the effects of antioxidant intake from a physiological viewpoint," Antioxidants, vol. 7, no. 9, p. 119, 2018.

[14] H. Sies, "Oxidative stress: a concept in redox biology and medicine,” Redox Biology, vol. 4, pp. 180-183, 2015.

[15] J. Peake, K. Nosaka, and K. Suzuki, "Characterization of inflammatory responses to eccentric exercise in humans," Exercise Immunology Review, vol. 11, pp. 64-85, 2005.

[16] M. C. Gomez-Cabrera, A. Salvador-Pascual, H. Cabo, B. Ferrando, and J. Vina, "Redox modulation of mitochondriogenesis in exercise. Does antioxidant supplementation blunt the benefits of exercise training?," Free Radical Biology \& Medicine, vol. 86, pp. 37-46, 2015.

[17] Z. Radak, K. Ishihara, E. Tekus et al., "Exercise, oxidants, and antioxidants change the shape of the bell-shaped hormesis curve," Redox Biology, vol. 12, pp. 285-290, 2017.

[18] K. Aoki, A. Nakao, T. Adachi, Y. Matsui, and S. Miyakawa, "Pilot study: effects of drinking hydrogen-rich water on muscle fatigue caused by acute exercise in elite athletes," Medical Gas Research, vol. 2, no. 1, p. 12, 2012.
[19] T. Kawamura, R. Fujii, K. Higashida, and I. Muraoka, "Hydrogen water intake may suppress liver glycogen utilization without affecting redox biomarkers during exercise in rats," Gazzetta Medica Italiana - Archivio per le Scienze Mediche, vol. 178, no. 9, pp. 611-617, 2019.

[20] S. Ohta, "Recent progress toward hydrogen medicine: potential of molecular hydrogen for preventive and therapeutic applications," Current Pharmaceutical Design, vol. 17, no. 22, pp. 2241-2252, 2011.

[21] T. Kawamura, Y. Gando, M. Takahashi, R. Hara, K. Suzuki, and I. Muraoka, "Effects of hydrogen bathing on exerciseinduced oxidative stress and delayed-onset muscle soreness," Japanese Journal of Physical Fitness and Sports Medicine, vol. 65, no. 3, pp. 297-305, 2016.

[22] T. Kawamura, K. Suzuki, M. Takahashi et al., "Involvement of neutrophil dynamics and function in exercise-induced muscle damage and delayed-onset muscle soreness: effect of hydrogen bath," Antioxidants, vol. 7, no. 10, p. 127, 2018.

[23] M. Yamazaki, K. Kusano, T. Ishibashi, M. Kiuchi, and K. Koyama, "Intravenous infusion of $\mathrm{H}_{2}$-saline suppresses oxidative stress and elevates antioxidant potential in thoroughbred horses after racing exercise," Scientific Reports, vol. 5, no. 1 , article $15514,2015$.

[24] J. E. Nogueira, P. Passaglia, C. M. D. Mota et al., "Molecular hydrogen reduces acute exercise-induced inflammatory and oxidative stress status," Free Radical Biology \& Medicine, vol. 129, pp. 186-193, 2018.

[25] C. Liu, R. Kurokawa, M. Fujino, S. Hirano, B. Sato, and X.-K. Li, "Estimation of the hydrogen concentration in rat tissue using an airtight tube following the administration of hydrogen via various routes," Scientific Reports, vol. 4, no. 1, article 5485, 2015.

[26] N. V. Margaritelis, V. Paschalis, A. A. Theodorou, A. Kyparos, and M. G. Nikolaidis, "Antioxidants in personalized nutrition and exercise," Advances in Nutrition, vol. 9, no. 6, pp. 813-823, 2018.

[27] V. Paschalis, A. A. Theodorou, A. Kyparos et al., "Low vitamin $C$ values are linked with decreased physical performance and increased oxidative stress: reversal by vitamin $\mathrm{C}$ supplementation," European Journal of Nutrition, vol. 55, no. 1, pp. 45-53, 2016.

[28] V. Paschalis, A. A. Theodorou, N. V. Margaritelis, A. Kyparos, and M. G. Nikolaidis, "N-Acetylcysteine supplementation increases exercise performance and reduces oxidative stress only in individuals with low levels of glutathione," Free Radical Biology \& Medicine, vol. 115, pp. 288-297, 2018.

[29] C. Yfanti, T. Åkerström, S. Nielsen et al., "Antioxidant supplementation does not alter endurance training adaptation," Medicine \& Science in Sports \& Exercise, vol. 42, no. 7, pp. 1388-1395, 2010.

[30] K. Higashida, S. H. Kim, M. Higuchi, J. O. Holloszy, and D. H. Han, "Normal adaptations to exercise despite protection against oxidative stress," American Journal of PhysiologyEndocrinology and Metabolism, vol. 301, no. 5, pp. E779E784, 2011.

[31] S. M. Ostojic, "Serum alkalinization and hydrogen-rich water in healthy men," Mayo Clinic Proceedings, vol. 87, no. 5, pp. 501-502, 2012.

[32] S. M. Ostojic and M. D. Stojanovic, "Hydrogen-rich water affected blood alkalinity in physically active men," Research in Sports Medicine, vol. 22, no. 1, pp. 49-60, 2014. 
[33] P. Drid, T. Trivic, C. Casals, S. Trivic, M. Stojanovic, and S. M. Ostojic, "Is molecular hydrogen beneficial to enhance postexercise recovery in female athletes?," Science \& Sports, vol. 31, no. 4, pp. 207-213, 2016.

[34] A. Da Ponte, N. Giovanelli, D. Nigris, and S. Lazzer, "Effects of hydrogen rich water on prolonged intermittent exercise," The Journal of Sports Medicine and Physical Fitness, vol. 58, no. 5, pp. 612-621, 2018.

[35] C. H. Ooi, S. K. Ng, and E. A. Omar, "Acute ingestion of hydrogen-rich water does not improve incremental treadmill running performance in endurance-trained athletes," Applied Physiology, Nutrition, and Metabolism, 2019.

[36] T. W. LeBaron, A. J. Larson, S. Ohta et al., “Acute supplementation with molecular hydrogen benefits submaximal exercise indices. Randomized, Double-Blinded, Placebo-Controlled Crossover Pilot Study," Journal of Lifestyle Medicine, vol. 9, no. 1, pp. 36-43, 2019.

[37] T. Mikami, K. Tano, H. Lee et al., "Drinking hydrogen water enhances endurance and relieves psychometric fatigue: a randomized, double-blind, placebo-controlled study," Canadian Journal of Physiology and Pharmacology, vol. 97, no. 9, pp. 857-862, 2019.

[38] M. Botek, J. Krejčí, A. J. McKune, B. Sládečková, and N. Naumovski, "Hydrogen rich water improved ventilatory, perceptual and lactate responses to exercise," International Journal of Sports Medicine, vol. 40, no. 14, pp. 879-885, 2019. 


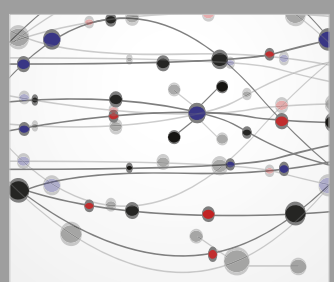

The Scientific World Journal
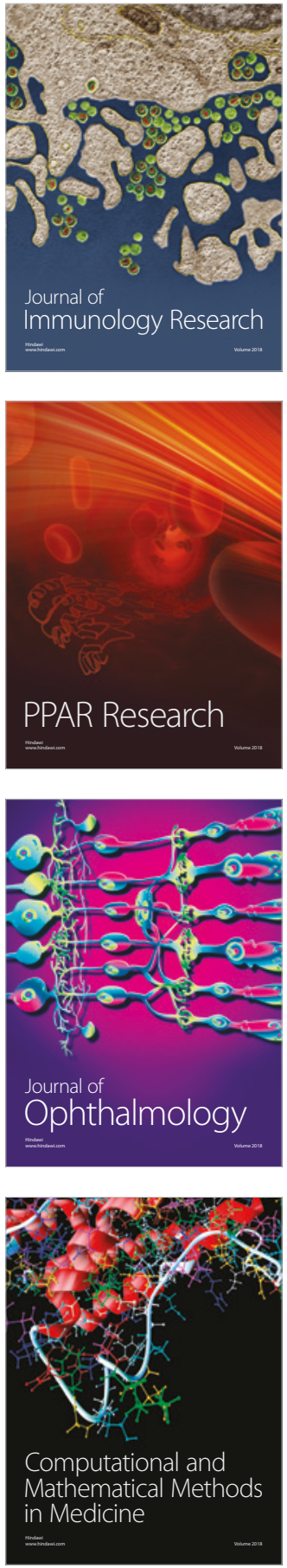

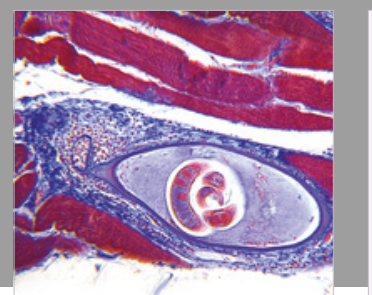

Gastroenterology Research and Practice

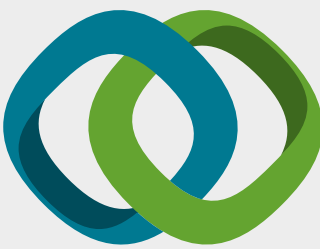

\section{Hindawi}

Submit your manuscripts at

www.hindawi.com
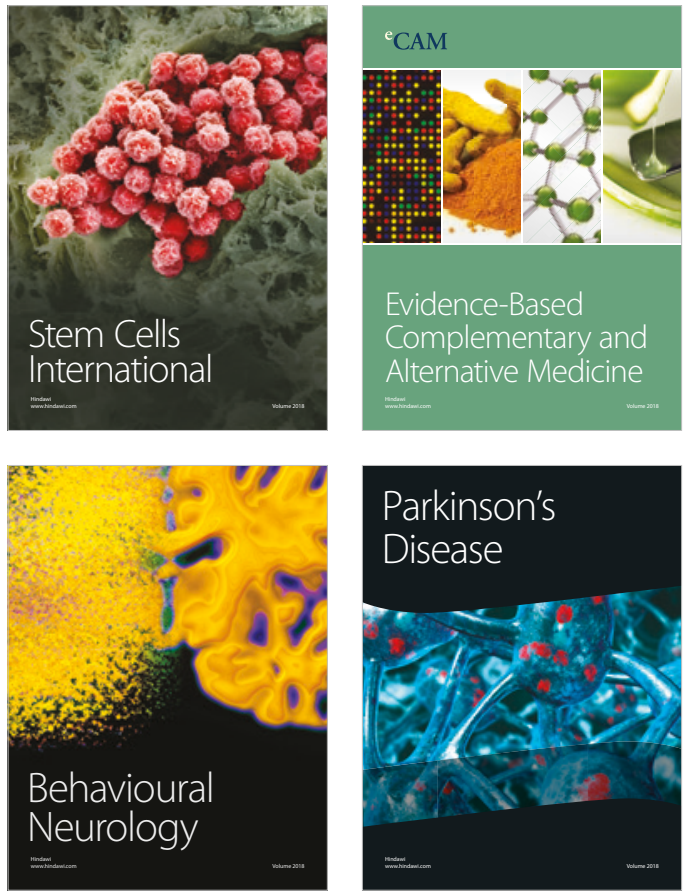

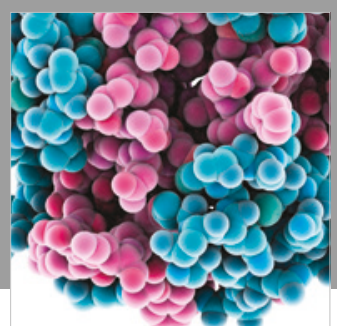

ournal of

Diabetes Research

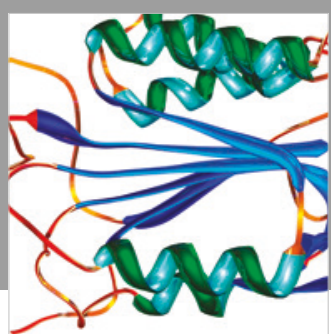

Disease Markers
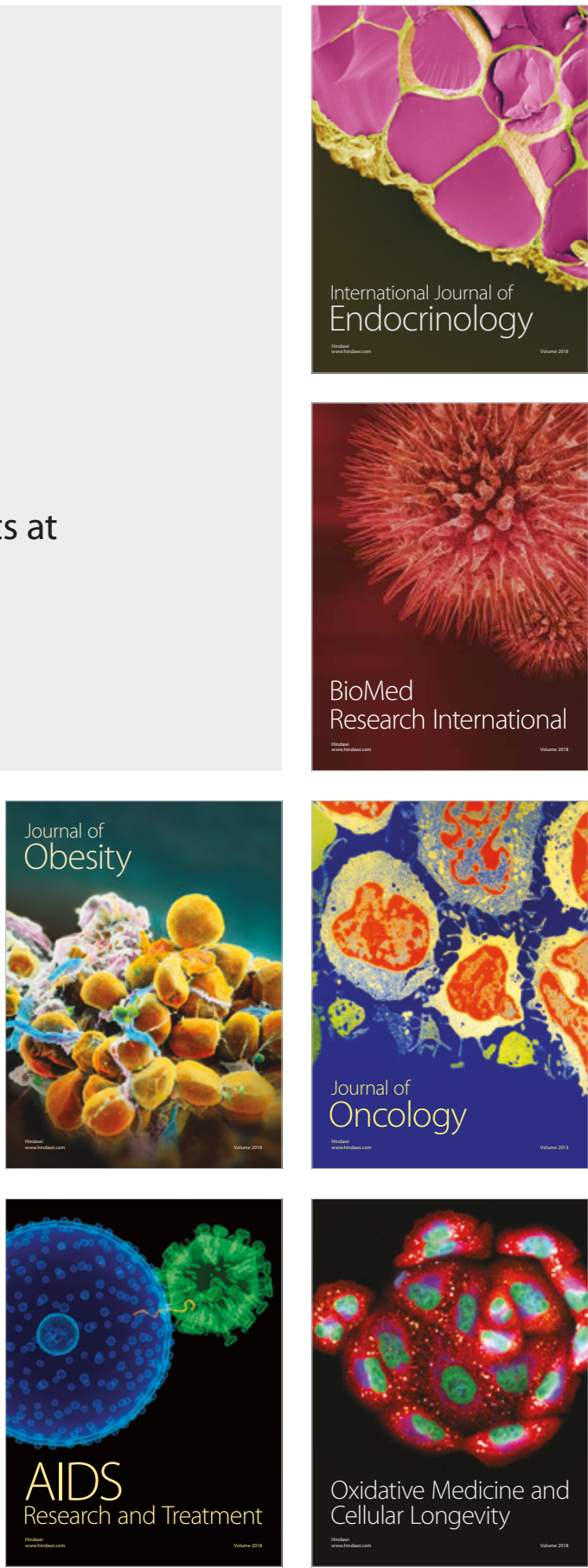